\title{
PRE-FREEZING AND PRE-MELTING
}

\author{
A. R. UbBelohde
}

Department of Chemical Engineering and Chemical Technology, Imperial College of Science and Technology, London, S.W.7, U.K.

When attempts are made to interpret the solid-liquid transition in terms of molecular structure, highly significant information can emerge from phemomena of pre-melting and pre-freezing. Empirically, on approaching the transition between solid and liquid, characteristic parameters of the phase under examination may show an accelerated rate of change, in the sense of a premonition of the change due to take place at the transition point itself. It is convenient to consider separately thermodynamic parameters such as the heat content, the volume, and derived quantities, and rate process parameters which have included critical shear strength and diffusion for the solid, and viscosity and other transport parameters for the melt. In certain cases many other methods have become available in recent years, especially in studying pre-melting.

Depending on the nature of the crystal, thermodynamic phenomena of pre-melting may be very prominent, or almost imperceptible. Prominent examples with various crystal structures include heat content and volume changes in polymethylene compounds ${ }^{1,}{ }^{2}$, heat content change of lithium fluoride $^{3}$ and alkali metals ${ }^{4}$, and thermal expansions of silver halides ${ }^{5}$. When only thermodynamic measurements are available, rigorous care is always necessary to eliminate a quite trivial effect known as " heterophase pre-melting". This arises from a small mole fraction $n / \mathcal{N}$ of impurity when this is not soluble in the crystals but is soluble in the melt. Theory shows that the excess $\Delta Q$ of volume or heat content change, which arises from actual formation of small amounts of liquid at a temperature $T$ below the limiting melting point $T_{\mathfrak{f}}{ }^{\circ}$, in such cases is related to the total change $\Delta Q^{\circ}$ by the equation

$$
\Delta Q^{\circ} / \Delta Q=\mathcal{N}\left(T_{f}^{\circ}-T\right) / n K
$$

where $K$ is the cryoscopic constant.

By graphical integration, $\Delta Q$ can be evaluated from a plot of $\mathrm{d} \Delta Q / \mathrm{d} T$. This permits two direct tests of heterophase pre-melting; if this predominates, a plot of $1 / \Delta Q$ against $T$ should give a straight line, and its slope $\mathcal{N} / n$ permits evaluation of the fraction of impurity causing heterophase pre-melting.

By these tests it is readily seen ${ }^{6}$ that the marked pre-melting of potassium thiocyanate, for example, cannot be due to hetero-impurities, since these would have to be present to the extent of 3.5-7 per cent to account for the pre-melting observed, and furthermore do not yield a straight-line plot. It is significant that a plot of $\log \Delta Q$ against $1 / T$ gives a straight line for this salt, suggesting that its pre-melting is associated with homophase lattice defects obeying a law

$$
n / \mathcal{N}=C \exp -E / R T
$$




\section{A. R. UBBELOHDE}

Its slope with $E \simeq 58 \mathrm{kcal} / \mathrm{mole}$ is suggestively near to the energy required to create lattice defects of about $47 \mathrm{kcal} / \mathrm{mole}$, as estimated from conductance measurements on the solid. Quite apart from these calculations, after careful purification any likely impurities are retained in solid solution and have a homophase effect on pre-melting, through the lattice defects they introduce. Such impurities can even be added deliberately; small amounts hardly affect the curves obtained ${ }^{1}$.

Another way of studying pre-melting where there is a marked change in heat content of the solid on approaching the melting point, is by precision cryoscopy. The solute added must not form solid solutions in the crystals. On progressive addition the freezing temperature falls, and the crystals separate with rapidly changing heat content; this becomes apparent by a changing cryoscopic constant. Tests on polymethylene hydrocarbons have been described by Oldham and Ubbelohde ${ }^{7}$, and on potassium thiocyanate by Rhodes and Ubbelohde ${ }^{8}$.

Non-thermodynamic techniques include ionic conductance and diffusion measurements on silver salts. Pre-melting effects run parallel with thermodynamic findings ${ }^{5}$. Optical measurements reveal pre-melting due to onset of rotation, for example in benzene ${ }^{9-11}$; dielectric measurements have been used to follow pre-melting in polymethylene ketones ${ }^{12}$. Ultra-violet absorption spectra confirm true homophase pre-melting of potassium thiocyanate $^{13}$. Mechanical effects include a marked decrease in the plasticelastic limit of cryolites on approaching their melting point, but theories underlying such effects have not yet attained sufficient confirmation to provide useful guides about structure.

All these premonitory changes in the solid state have no obvious explanation in terms of the classical Phase Rule concept of melting as a transition between completely independent solid and liquid phases. The changes resemble $\Lambda$-point phenomena in solids, which have been given an interpretation in terms of " smearing" of the transformation and the co-existence of domains of alternative structure in hybrid crystals ${ }^{14-16}$. Related to this is an early theory about "heterophase fluctuations"17. However, two important considerations about melting make its relationship with a $\Lambda$-point transformation only rather superficial:

(i) In all known cases, even when premonitory effects constitute a substantial fraction of the total change on passing from the solid well below the melting point, to the melt, there always is a discontinuous jump from solid to liquid at a melting point in properties such as the density. In other words, melting always is a discontinuous phase change, even if the two equations of state begin to approximate by a rapid change of slope before they actually intersect.

It seems possible that at sufficiently high pressures a continuous transition from solid to liquid might be attained. Theoretical arguments for and against a critical point for solid-liquid, by analogy with the well known critical point for solid-gas, have not yet reached finality ${ }^{18}$. The existence of marked pre-melting is an important consideration in such cases.

(ii) In ordinary $\Lambda$-point phenomena, on both sides of the peak the crystals show long-range order; in the transition region the solid is a hybrid of domains of both kinds. However, since liquids do not show long-range 
order, "heterophase fluctuations" involving a hybrid of solid and liquid offer no advantage as a model to explain pre-melting over the concept of " co-operative defects" in the crystals. For co-operative defects the energy of formation no longer remains independent of neighbouring defects, because their concentration is too large. For examples in relation to premelting see Oldham and Ubbelohde ${ }^{7}$; there are no doubt many other types.

When pre-melting is due to the onset of "rotation" of some kind in the crystal, as may be the case for many molecular crystals, suitable additives in solid solution can lower the potential barriers hindering this disorder and thus enhance the pre-melting. Tests on benzene crystals have been described by Thompson and Ubbelohde ${ }^{19}$.

\section{PRE-FREEZING IN MELTS}

By analogy, the explanation of pre-melting as arising from co-operative defect formation in the crystals suggests that in liquids pre-freezing may involve incipient development of long-range order of some kind. Such incipient ordering of the molecules is conveniently described as " clustering ", and is likely to be favoured by certain molecular shapes, such as rods or plates. Most important is the fact that in pre-melting, the co-operative defects arise in a specific crystal structure. On the other hand, clustering of molecules in a melt can involve various alternative forms of incipient ordering. Usually no kinetic obstacles prevent all possible types of clustering from being realized simultaneously in the melt when the temperature is sufficiently low. On this model of a melt, pre-freezing involves increasing concentrations of the whole diversity of realizable clusters, dissolved in monomer. Transport properties of melts can give a sensitive indication of the presence of such clusters. For example, some polyphenyl molecules ${ }^{20}$ such as $p$-terphenyl give melts with excellent straight-line polts of the relationship

$$
\log \eta=A+B / T
$$

where $\eta$ is the viscosity.

Other closely related polyphenyl hydrocarbons such as 0 -terphenyl give evidence of very extensive cluster formation as the freezing point is approached and readily supercool to extremely viscous melts ${ }^{21}$. Extensive interlocking of the molecules into clusters accounts for the pre-freezing. When the liquid flows, these clusters behave like colloidal particles and the viscosity $\eta$ can be written according to the Einstein formula

$$
\eta / \eta_{\mathrm{si}}=1+2 \cdot 5 \Phi+7 \Phi^{2} \ldots
$$

where $\eta_{\text {si }}$ is the hypothetical viscosity, calculated by extrapolation, which the liquid would have if it still consisted wholly of monomers, and $\Phi$ is the volume fraction of the melt occupied by the clusters ${ }^{21}$. Figure 1 gives the fraction $\Phi$ for $o$-terphenyl calculated on this basis, from the experimental viscosity data.

Plots of specific volume of such melts against temperature show that the liquid supercools and readily passes into a glass. Since this evidence shows that spontaneous nucleation is practically absent in the supercooled melt, 
A. R. UBBELOHDE

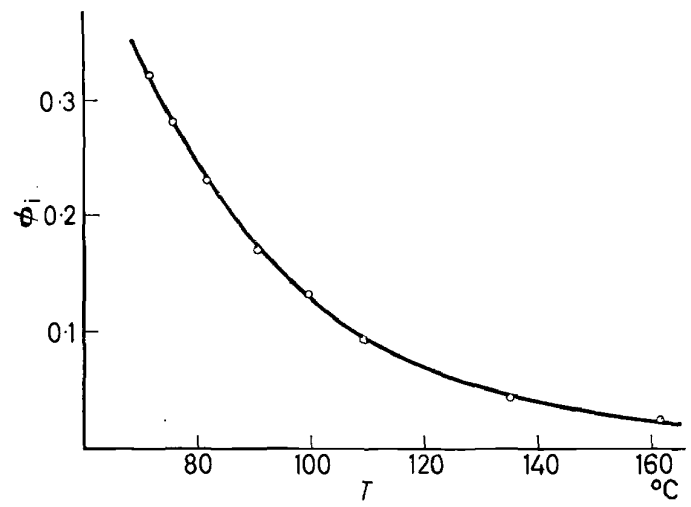

Figure 1. Cluster formation in o-terphenyl21. (By courtesy of the Faraday Saciety)

practically none of these clusters can have the structure of crystal nuclei capable of giving long-range order in three dimensions. This can be readily understood in the light of the well-known theory of Volmer, Becker and Doring about the concentration of crystal nuclei in a melt, provided allowance is made for the fact that there can be many alternative forms of cluster packing which are incapable of indefinite extension in three dimensions. Even with simpler quasi-spherical molecules, non-crescible modes of clusterpacking can be important in the melts as the freezing points are approached (cf. Frank, references in Ubbelohde ${ }^{14-16}$ ).

Though transport properties other than the viscosity can be likewise sensitive to cluster formation, they have been much less investigated; the thermal conductivity of some $n$-polymethylene hydrocarbons indicates marked pre-freezing phenomena (Figure 2).

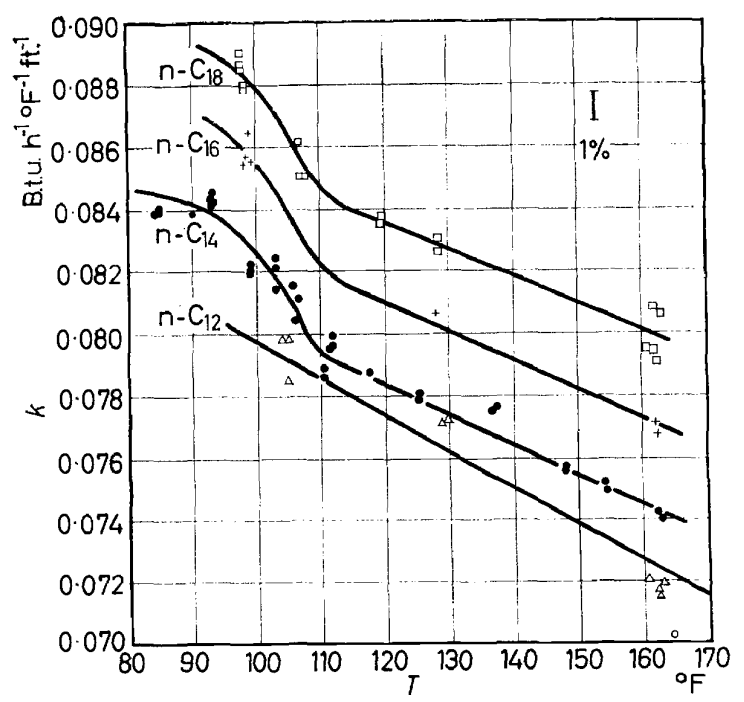

Figure 2. Thermal conductivity of some n-polymethylene hydrocarbons. (By courtesy of the American Institute of Chemical Engineers) 


\section{PRE-FREEZING AND PRE-MELTING}

\section{References}

1 A. R. Ubbelohde. Trans. Faraday Soc., 34, 282, 292 (1938)

2 A. Van Hook and L. Silver. 7. Chem. Phys., 10, 686 (1942)

s T. B. Douglas and J. L. Dever. F. Am. Chem. Soc., 76, 4826 (1954)

4 L. G. Carpenter. 7. Chem. Phys., 21, 2244 (1953)

5 A. W. Lawson. Phys. Rev., 78, 185 (1950)

6 D. W. Plester, S. E. Rogers and A. R. Ubbelohde. Proc. Roy. Soc. (London), A, 235, 469 (1956)

7 J. W. H. Oldham and A. R. Ubbelohde. Proc. Roy. Soc. (London), A, 176, 50 (1940)

8 E. Rhodes and A. R. Ubbelohde. Trans. Faraday Soc., 55, 1705 (1959)

- A. Rousset. Compt. rend., 219, 485, 546 (1944)

${ }^{10}$ E. R. Andrew and R. G. Eades. Proc. Roy. Soc. (London), A, 218, 537 (1953)

11 A. Frubling. Ann. Phys., 6, 401 (1951)

12 A. Müller. Proc. Roy. Soc. (London), A, 174, 137 (1940)

13 E. Rhodes and A. R. Ubbelohde. Proc. Roy. Soc. (London), A, 251, 156 (1959)

14 A. R. Ubbelohde. Brit. 7. Appl. Phys., 7, 313 (1956)

15 A. R. Ubbelohde. "Report on Melting and Grystal Structure", F. Roy. Coll. Sci., 26, 1 (1956)

16 A. R. Ubbelohde. Quart. Rev. (London), 11, 246 (1957)

${ }^{17} \mathrm{~J}$. Frenkel. Kinetic Theory of Liquids, Oxford University Press (1946)

18 A. R. Ubbelohde. Quart. Rev. (London), 4, 367 (1950)

19 F. W. Thompson and A. R. Ubbelohde. Trans. Faraday Soc., 46, 349 (1950)

20 J. N. Andrews and A. R. Ubbelohde. Proc. Roy. Soc. (London), A, 228, 435 (1955)

21 E. McLaughlin and A. R. Ubbelohde. Trans. Faraday Soc., 55, 28 (1959)

22 B. C. Sakiadis and J. Coates. A. I. Ch. E. Journal, 3, 123 (1957) 